\title{
CORRESPONDENCE
}

\section{STRUCTURES DATA SHEETS}

The following note has been received from Dr. J. H. Argyris and Mr. P. C. Dunne on the use of the sheets recently issued as Volume III of the Structures Data Sheets, which were prepared when they were members of the Society's Technical Staff. It is felt that this note will be of interest to many users of the data sheets and it is proposed that a reprint of this note will be sent for insertion in all sets of the sheets.

THE volume attempts to give in data sheet form a rational prociedure for stressing conical 1 tubes under an arbitrary distribution of transverse forces. Since the data sheets are intended for use in wing stressing, at first sight they may appear to be much more restricted in their application than may reasonably be expected from such a large number. It is hence desirable to list some of the main assumptions on which this work is based, together with the considerable possibilities of extending usefully its range.

\section{MAIN ASSUMPTIONS AND POSSIBILITIES OF RELAXING THEM}

1 (a) The structure consists of conical segments stiffened transversely by a closely spaced system of ribs rigid in their own plane but perfectly flexible normal to it.

(b) The taper or sweepback should be such that no generator is inclined to the normal to the ribs by more than $10^{\circ}$.

1 (a) The assumption that the ribs completely prevent cross-section distortion but do not impede warping does not affect the calculation of the engineers' theory and Bredt-Batho stresses. In general, the effect of rib deformability is only important in regions of large concentrated loads or pronounced changes of cross section (e.g. at cut-outs). Moreover, in closed segments of wings with conventional plate or girder ribs the axial constraint stresses are usually conservative when based on the rigid rib assumption. Since rib deformability reduces the resistance to warping of a closed segment the disturbance to the stress distribution at the join between an open and a closed segment will be its most marked effect: this is generally most adverse on the open segment.

(b) The theory may be applied to swept-back tubes with large angles of sweep, provided that they are of the type with ribs normal to the mean sweepback line. A special calculation will be necessary to estimate the warping characteristics of the carry-through bay connecting the port and starboard wings. The detailed stress distribution in the central part of the wing where there is a sudden change in the direction of the ribs (root triangle) remains as a complicated problem. One limitation of the applicability of the theory to swept-back tubes is that the effect of rib deformability is much more pronounced.

For swept-back wings with ribs along the line of flight, or wings with very large taper (delta-wings), the theory is not applicable. It should be noted, however, that for wings with large mean sweepback angle $\alpha$ and small taper the engineers' theory and Bredt-Batho stress system are statically consistent. This presumes that the bending moments are calculated about axes parallel to the ribs and factored by $\sec \alpha$ and that the shear flows are regarded as acting along trapezoidal elements.

2 (a) The tube consists of four direct stress-carrying booms and a number of purely shearcarrying walls.

(b) The thicknesses of all walls are constant lengthwise and the areas of the booms are proportional to the distance from the apex of the cone.

2 (a) The restriction on the number of direct stress-carrying booms is without any special significance when calculating the engineers' theory stresses. All formula given in the appropriate data sheets may be generalised immediately for an $n$-boom tube by changing the summation sign $\Sigma_{1}^{4}$ to $\sum_{1}^{n}$. Furthermore, the effect of a continuous direct stress-carrying skin may be included by changing the summation formulæ into integral ones. The four-boom approximation will usually be found sufficient for the calculation of the 
torsion-bending stresses in an open tube; a more elaborate analysis, based on the $n$-boom conception, may be found in Section 6.5 .7 of Reference 1 .

The calculation of the axial constraint stresses for tubes with more than four booms, on an exact basis, is a tedious procedure and would probably not be suitable for presentation in data sheet form. Note, however, that the large number of diagrams giving the longitudinal variation of the axial constraint stresses are immediately applicable to conical tubes with any number of booms, so long as the longitudinal variation of booms and wall thicknesses follows assumption $2(b)$. Furthermore, from the practical point of view the four-boom approximation is often sufficient for a reasonable estimate of the axial constraint or shear lag stresses, so long as the effective boom areas are properly estimated and the cross-sections considered are not at, or near, a cut-out. Now, the most important stressing problem at cut-out is the design of the proper reinforcement of the spar booms and in the Appendix to Part VI (Ref. 1) the authors show how this can be done simply on the basis of a preliminary four-boom analysis by the use of two additional diffusion data sheets only. There will also be found a method of obtaining the effective boom areas for the four-boom approximation to the axial constraint stresses.

(b) If the boom areas and wall thicknesses are similarly distributed at all cross sections, but their longitudinal variations do not follow the simple laws postulated under $2(b)$, a considerable number of the present data sheets may still be applied with very minor or no alterations. This, for example, is true for the engineers' theory, Bredt-Batho, and torsion-bending stresses and for the cross-wise variation of the axial constraint stresses. Only the longitudinal variation of the latter stresses (i.e. the $G$-functions) takes a different analytical form but in most cases it should still be possible by suitable idealisation of the longitudinal variation of booms and wall thicknesses to make good use of the present data sheets.

If the distribution of the direct and shear stress-carrying material is not similar at all cross sections it is, in principle, still possible by suitable idealisation to obtain a structure following the laws of variation assumed in these data sheets. This method is reasonably reliable in many cases, but it appears preferable when finding the engineers' theory and Bredt-Batho stresses and the axial constraint torque, to omit the previous step in the idealisation and to use a different analysis, also described in detail in the Appendix to Ref. 1. It is important to realise that in tubes with non-similar distribution of material there is no flexural axis in the strict sense of that existing in idealised tubes, and furthermore, there is no zero-warping axis. The analysis proceeds as follows: At different stations the boom load gradients $d P_{\mathrm{E}} / d z$ are calculated by the engineers' theory. The shear flows corresponding to zero rate of twist at all cross sections are then determined from formulæ which may be obtained from the equations for the engineers' theory shear flows given in the data sheets by substituting $-d P_{\mathrm{E}} / d z$ for $\left(\bar{Q}_{\mathrm{y}} / I_{\mathrm{x}}\right) B \bar{y}$ (or $\left(\bar{Q}_{\mathrm{x}} / I_{\mathrm{y}}\right) B \bar{x}$ ). The torque of the zero twist shear flows about any convenient axis through the apex, is then subtracted from the torque of the external load system, about the same axis, to give the torque from which the Bredt-Batho shear flows are calculated.

It is shown in the Appendix to Ref. 1 (Section A.2.1) how the torque from which the axial constraint stresses arise can be calculated very easily. To find their distribution, however, it will still be necessary to use an idealised tube structure (or a series of segments) following the longitudinal variation postulated in the present data sheets. If the idealised structure consists of a single conical tube it is best to make the tubes most similar at the cross sections where the axial constraint stresses are most marked, e.g. at a root if built in. When for greater accuracy the structure is divided into a number of idealised conical segments, in general, it is preferable to make the actual and idealised cross sections identical in the middle of each segment. An exception to this rule is advisable at stations with peaks in the axial constraint stresses where the previous procedure of making the two respective cross sections identical should be used. The problem of designing the spar booms at, or near, cut-outs may be treated as indicated at the end of $2(a)$.

In the case of non-conical tubes, the method of the last paragraph may still be applied, in principle. It should be noted that for the calculation of the engineers' theory and Bredt-Batho stresses the actual structure is used, but when finding the torque to be carried by the latter stresses, allowance must be made for the torque arising from the boom loads. The torque giving rise to the axial constraint stresses may be calculated approximately by the same procedure as advised in the last paragraph for conical tubes. The longitudinal variation of the axial constraint boom loads may be estimated by considering the structure as a conical tube, or a series of conical segments, with the 
same longitudinal variation of the material as assumed in the present data sheets. However, the transverse variation of the boom loads must be based on the current cross-section. The shear flows in the walls are then calculated up to a constant from the boom load gradients. Finally, the unknown constant is determined from the condition of zero torque due to axial constraint boom loads and shear flows (See also Ref. 2).

\section{SUGGESTED STRESSING SEQUENCE}

Following this discussion of the assumptions it may be worth while to point out that essentially any stressing procedure is based on an idealisation of the geometry of the structure and the behaviour of the material. Considerable physical insight is often required in finding the most suitable idealised structure, both from the accuracy and computational point of view. In most cases a cruder idealisation is satisfactory for finding the axial constraint stresses than for the engineers' theory stresses. Bearing this in mind, it is suggested that the calculations should proceed in the following stages:-

(I) Calculate the engineers' theory and Bredt-Batho boom loads and stress flows in a suitably idealised structure; this structure need not necessarily be four-boomed (see 2(a) above). Or alternatively find the statically equivalent stress system in the actual structure following the procedure given in 2(b). (See also Section 15.9 of Ref. 2.)

(II) From the boom loads and stress flows found by the first method of stage (I) calculate the direct and shear stresses in the actual structure. This step is obviously redundant when the actual structure is used in step (I).

(III) Calculate the axial constraint (or torsion-bending) boom loads and shear flows in a suitable four-boom idealisation (see $2(a)$ and $2(b)$ above). Convert these boom loads and shear flows into a stress distribution in the actual structure.

(IV) Obtain the total stresses by adding those of stages (II) and (III). Note that if the two idealised structures are identical it is better to sum the boom loads and stress flows of stages (I) and (III) and thence the total stresses in the actual structure.

(V) Correct, if necessary, for diffusion effects at cut-outs (again see 2(a)). It may sometimes be necessary to correct also for the small out-of-balance boom loads that will arise at the join of two segments when the idealisation of stage (I) differs from that of stage (III).

\section{REFERENCES :}

1. Hadj-Argyris, J. and DunNe, P. C. The general theory of cylindrical and conical tubes under torsion and bending loads.

Parts I-IV Journal of the Royal Aeronautical Society, February 1947.

Part V Journal of the Royal Aeronautical Society, September and November 1947.

Part VI Journal of the Royal Aeronautical Society, May and June 1949.

2. Hadi-Argyris, J. and Dunne, P. C. Structural Analysis, Part 2 of Structural Principles and Data. Pitman, London, 1952.

J. H. Argyris (Associate Fellow).

P. C. Dunne (Associate Fellow). 